\title{
EFFECT OF NON-SURGICAL PERIODONTAL THERAPY ON SERUM AND CREVICULAR LEVEL OF ADVANCED GLYACATION END PRODUCT IN CHRONIC PERIODONTITIS AMONG A SAMPLE OF THE EGYPTIAN POPULATION
}

\author{
Wesam Abdel Moneim *, Amira Maged * and Olfat G. Shaker*
}

\begin{abstract}
Background: AGEs are formed under oxidative conditions and are called advanced glycoxidation end products. Periodontitis is a multifactorial inflammatory condition. The periodontal tissue damage is caused directly by bacteria and indirectly by inflammation and immunologic response against the bacteria. This inflammatory response is characterized by generation of prolonged release of neutrophil proteolytic enzymes, pro-inflammatory mediators and reactive oxygen species (ROS). Therefore, periodontitis is strictly linked to numerous pathologies which are related to production of oxidative stress and AGEs.
\end{abstract}

Methods: The study was conducted on thirty participants, they were divided into two groups; group (A) which included fifteen medically free subjects, and group (B) which included fifteen patients suffering from periodontitis. Serum and GCF samples were collected for assessment of AGEs before and after non -surgical periodontal therapy. Data were analysed by SPSS.

Results: Periodontitis group showed statistically significant higher mean AGE levels than control group in both serum and GCF samples. Moreover, there was a statistically significant decrease in AGE levels in both serum and GCF after treatment.

Conclusion: Crevicular level of AGEs can be used as a non-invasive diagnostic and prognostic biomarker for periodontitis.

KEYWORDS: advanced glycation end products, chronic periodontitis, GCF

\section{INTRODUCTION}

Advanced glycation end products are a complex and heterogeneous group of compounds which are produced by the non-enzymatic and spontaneous reactions of the carbonyl group of carbohydrates with proteins, nucleic acids or lipids through a series of reactions. Actually, this process is composed of two steps: i) The condensation of a sugar aldehyde with

* Associate professor in Department of Oral Medicine and Periodontology, Faculty of dentistry, Cairo University.

** Professor in Department of Biochemistry, Faculty of Medicine, Cairo University. 
a free amino group of proteins (usually hydroxyllysine or arginine residues) forming a non-stable Schiff base ii) Formation of Amadori products from Schiff base. This glycation process, which is also known as the Millard action, was described in the early 1912's by Louis-Camille Maillard. The Schiff base and especially Amadori products may undergo oxidation, dehydration, and polymerization to rise other AGEs. The AGEs that formed under oxidative conditions are called advanced glycoxidation end products (Goldin et al, 2006).

Chronic disorders are significantly accompanied with oxidative stress in humans and animals, as decreased expression of anti-oxidative enzymes along with NADPH oxidase activation, all contribute to the high production of ROS in the tissues. Activation of nuclear factor (NF)- $\chi \mathrm{B}$, and JNK pathways is accompanied by obesity, high-fat diet, and cellular stresses (ROS), which have the ability to increase the inflammatory responses in body tissues. Some ligands including TNFR, IL-1R, and RAGE that are receptors for TNF- $\alpha$, IL-1, and AGEs can also activate the JNK pathway during metabolic dys-regulation (Shoelson et al, 2006). These processes that are occurred in diseases are the principal causative factors for the formation of AGEs (Elaheh et al, 2018).

Periodontitis is considered as a multifactorial condition that is caused by infection with pathogenic bacteria and significantly modified by a variety of environmental and genetic factors. The periodontal tissue damage is caused directly by bacteria and indirectly by inflammation and immune response against bacteria (McDevitt et al, 2000).

According to Chapple and Matthews (2007), this hyper inflammatory response fails to remove the causative pathogens and also generates a prolonged release of neutrophil proteolytic enzymes, pro-inflammatory mediators and reactive oxygen species (ROS), which in turn destroy the periodontal attachment. Fusobacterium nucleatum and periodonto-pathogens can induce an increased intracellular production of ROS in neutrophils along with their release.

Therefore, periodontal diseases are strictly linked to numerous pathologies related to the production of oxidative stress (Szczepanski et al,2007) and AGEs (Allen et al, 2009).

Accordingly, the present study was conducted to find out the role of AGEs and the valuable use of its crevicular level over the serum level in the diagnosis and prognosis of periodontitis after nonsurgical periodontal therapy.

\section{SUBJECTS AND METHODS}

Thirty participants, 21 females, and 9 males (ranging in age from 35-60) years were included in the study. They were selected from outpatient clinic of Oral Medicine, and Periodontology Department, Faculty of Dentistry, Cairo University. The study was conducted from Jan 2015 to Sep 2017.

They were divided to the following two groups:

Group (1): included 15 healthy participants free of any systemic diseases according to the modification of Cornell medical index (Abramson JH 1966.)

Group (2): included 15 patients of periodontitis and free of any other systemic diseases according to Cornell medical index (Abramson JH 1966.)

All participants were informed of the objectives and nature of the study and showed willingness to come for the follow up visits till the end of the study. They were given written approval consent to sign.

\section{Inclusion criteria}

Age $\geq 35$ years and a minimum of 20 natural teeth, not including third molars

As for patients with periodontitis, participants with moderate to severe periodontitis having gingival index GI $\geq 1$, probing depth $\mathrm{PD} \geq 5$, and clinical attachment level CAL $\geq 4 \mathrm{~mm}$, and bone loss affecting $>30 \%$ of existing teeth on clinical/ 
radiographic examination according to the American academy of periodontology 2000 were included in the study.

\section{Exclusion criteria}

Periapical pathologies, exposure to mechanical force as a result of occlusion/ orthodontics, and any systemic disease such as, human immunedeficiency virus, cancer, diabetes mellitus, or any other diseases which may affect the biomarkers levels and the periodontal conditions, smoking over the past 5 years, exposure to steroid therapies, radiation/immune-suppressive therapies, allergic reaction to any kind of drugs. Participants featured no history of either periodontal or drug therapies within the preceding 6 months, such as antibiotic courses, anti-inflammatory treatments, or other pharmacological treatments.

\section{Periodontal examination}

At baseline, all participants were subjected to a full- mouth examination. For periodontitis patients, clinical parameters were recorded, including (plaque index PI, GI, PD, and CAL). Again, these parameters were recorded 3 months following scaling and root planning (SRP) for group 2 participants.

PD and CAL were measured using a Williams's periodontal probe (Hu-Friedy, Chicago, IL, USA). Individuals were assessed twice with a 48-h interval. If the difference between the two measurements fall within a $<10 \% \mathrm{~mm}$ range, then researcher measurements were considered to be acceptable (Schwarz et al, 2006).

\section{Periodontal treatment}

Once baseline crevicular samples were taken, individuals with periodontitis were subjected to non-surgical periodontal treatment, SRP using manual scalers and curettes with local anaesthesia. Periodontal treatment was completed in 2-3 visits for 2 weeks according to each patient's requisites, and each visit was 45-60 min long. No pharmacological interventions were prescribed as part of the course of treatment; they only were given oral hygiene instructions, using the modified Bass approach, toothpaste, and interdental tools.

\section{Samples collection and analysis}

\section{1- Site Selection and GCF Collection:} Clinical and radiologic examinations and sampling site selections were conducted by a single examiner. Samples were gathered the day after patients had been given clinical assessment. Thus to avoid mixing GCF and blood associated with the probing at inflamed areas. Two sites per individual were chosen from each group. GCF samples were taken from mesio-buccal or disto-buccal sites outside of single-rooted teeth. Samples were taken at baseline from the two groups and 6 weeks after SRP for periodontitis group. For periodontitis group; GCF sample was taken from the areas with the most prominent clinical indications of inflammation (the highest GI score and BOP) and the highest PD with radiographic readings of bone loss. For healthy groups, GCF samples were acquired from areas that showed no clinical inflammation $(\mathrm{GI}=0$, without BOP). Before crevicular sampling, all supra gingival plaque was eliminated from the sample area with a sterile cotton roll. The site was then washed with water then sectioned off with cotton balls, and gradually air-dried to avoid salivary contamination. Paper strips were positioned inside the crevice until a small amount of resistance was encountered then allowed to remain in place for 30 seconds (Griffiths, 2003). This process was conducted very gently to prevent manual damage to gingival tissues. The degree of GCF on the strips was calculated by weighing the collected liquid. The strips were put into closed and numbered plastic micro-centrifuge cases, and then liquid was weighed again, immediately after collection, to take evaporation into account (Tu"ter et al, 2010). Samples contaminated with blood and saliva were disregarded. Two strips from each study participant were put into a single (coded) Eppendorf container and combined to form one sample, and quickly 
stored at $-80 \mathrm{C}$ until they could be evaluated.

\section{2-Serum collection}

Venous samples were obtained to measure the serum level of AGEs using the ELISA technique after $12 \mathrm{~h}$ of fasting. Blood samples were collected in sterile tubes and allowed to clot at room temperature. Sera were isolated by centrifugation and stored frozen below -20 until assayed for the AGEs level.

\section{Measurement of AGE level in GCF:}

The level of AGE was measured using the sandwich Enzyme-Linked Immunosorbent Assay (ELISA) kit. The kit was provided by Bioassay Technology Laboratory, Shanghai, CHINA. AGEs was added to the wells which were pre-coated with AGEs monoclonal antibody. Then, after cubation, a biotin-conjugated anti-human AGEs antibody was added to bind to human AGEs. After incubation, unbound biotin-conjugated anti-human AGEs antibody was washed away during a washing step. Next step was addition of Streptavidin-HRP to bind to the biotin-conjugated anti-human AGEs antibody. After incubation unbound Streptavidin-HRP was washed away during a washing step. The Substrate solution was then added and the color developed in proportion to the amount of human AGEs. Finally, the reaction was terminated by addition of acidic stop solution and absorbance was measured at $450 \mathrm{~nm}$.

\section{Statistical analysis:}

Numerical data were explored for normality by checking the distribution of data and using tests of normality (Kolmogorov-Smirnov and Shapiro-Wilk tests). Age, Pocket Depth (PD), Clinical Attachment Level (CAL), serum and GCI Advanced Glyacation product (AGE) levels data showed normal (parametric) distribution while Gingival Index (GI) and Plaque Index (PI) data showed non-normal (nonparametric) distribution. Parametric data were presented as mean, standard deviation (SD) and 95\% Confidence Interval (95\% CI) for the mean values while non-parametric data were presented as median and range values. For parametric data, Student's t-test was used to compare between age values in the two groups. Paired t-test was used to study the changes after treatment in PD and CAL. Repeated measures ANOVA test was used to compare between AGE levels in the two groups as well as the changes within each group. Bonferroni's post-hoc test was used for pair-wise comparisons when ANOVA test is significant. Pearson's correlation coefficient was used to determine the correlation between PD, CAL and AGE levels. For non-parametric data; Wilcoxon signed-rank test was used to study the changes after treatment in GI. Spearman's correlation coefficient was used to determine the correlation between GI and AGE levels. Qualitative data were presented as frequencies and percentages. Fisher's Exact test was used for comparisons between the groups. The significance level was set at $\mathrm{P} \leq 0.05$. Statistical analysis was performed with IBM SPSS Statistics for Windows, Version 23.0. Armonk, NY: IBM Corp.

\section{Demographic data:}

There was no statistically significant difference between mean age values in the two groups. There was also no statistically significant difference between gender distributions in the two groups.

TABLE (1) Mean, standard deviation (SD), frequencies (n), percentages and results of Student's t-test and Fisher's Exact test for comparisons of demographic data in the two groups

\begin{tabular}{cccc}
\hline & $\begin{array}{c}\text { Periodontitis } \\
(\mathrm{n}=15)\end{array}$ & $\begin{array}{c}\text { Control } \\
(\mathrm{n}=15)\end{array}$ & $P$-value \\
\hline Age (Years) & & & \\
Mean (SD) & $50.5(6)$ & $49.8(5.6)$ & 0.799 \\
\hline $\begin{array}{c}\text { Gender [n (\%)] } \\
\text { Male }\end{array}$ & $5(33.3)$ & $4(26.7)$ & 1.000 \\
Female & $10(66.7)$ & $11(73.3)$ & \\
\hline
\end{tabular}

*: Significant at $P \leq 0.05$ 


\section{Clinical parameters}

\section{Changes after treatment in Periodontitis group}

There was a statistically significant decrease in Probing Depth (PD) $(P$-value $<0.001$, Effect size $=$ 1.629), Clinical Attachment Level (CAL) ( $P$-value $<0.001$, Effect size $=1.927)$, Plaque Index $(\mathrm{PI})$ $(P$-value $=0.024$, Effect size $=0.800)$ and Gingival Index $(\mathrm{GI})(P$-value $=0.010$, Effect size $=0.914)$.

\section{Advanced Glyacation product (AGE) levels}

\section{Comparison between the two groups}

In serum either before or after treatment, Periodontitis group showed statistically significantly higher mean AGE levels than control group ( $P$-value
$<0.001$, Effect size $=0.709)$ and $(P$-value $=0.014$, Effect size $=0.361)$, respectively.

Similarly in GCF either before or after treatment, Periodontitis group showed statistically significantly higher mean AGE levels than control group ( $P$-value $<0.001$, Effect size $=0.683)$ and $(P$-value $=0.007$, Effect size $=0.414)$, respectively.

\section{Changes after treatment in Periodontitis group}

Whether in serum or GCF, there was a statistically significant decrease in AGE level after treatment $(P$-value $<0.001$, Effect size $=0.775)$ and $(P$-value $<0.001$, Effect size $=0.785)$, respectively .

TABLE (1) Descriptive statistics and results of paired t-test and Wilcoxon signed-rank test for comparison between clinical parameters before and after treatment within Periodontitis group

\begin{tabular}{lcccc}
\hline Clinical parameters & $\begin{array}{c}\text { Before treatment } \\
(\mathrm{n}=15)\end{array}$ & $\begin{array}{c}\text { After treatment } \\
(\mathrm{n}=15)\end{array}$ & P-value & Effect size \\
\hline PD [Mean (SD)] & $4.63(0.74)$ & $3.25(0.89)$ & $<0.001^{*}$ & $d=1.629$ \\
CAL [Mean (SD)] & $4.88(0.64)$ & $3.5(0.76)$ & $<0.001^{*}$ & $d=1.927$ \\
GI [Median (Range)] & $2.5(2-3)$ & $1(1-1)$ & $0.010^{*}$ & $r=0.914$ \\
PI [Median (Range)] & $2(1-3)$ & $0.5(0-2)$ & $0.024^{*}$ & $r=0.800$ \\
\hline
\end{tabular}

*: Significant at $P \leq 0.05$

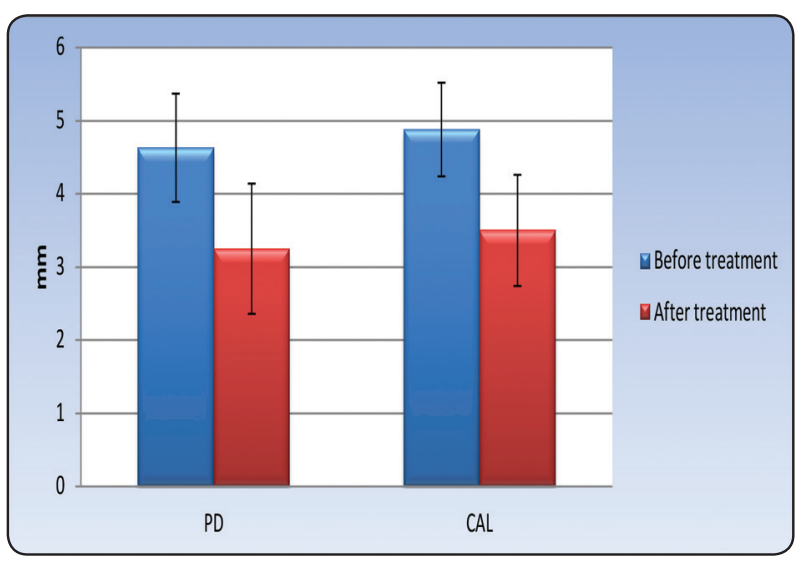

Fig. (1) Bar chart representing mean and standard deviation values for Pocket Depth (PD) and Clinical Attachment Level (CAL) before and after treatment

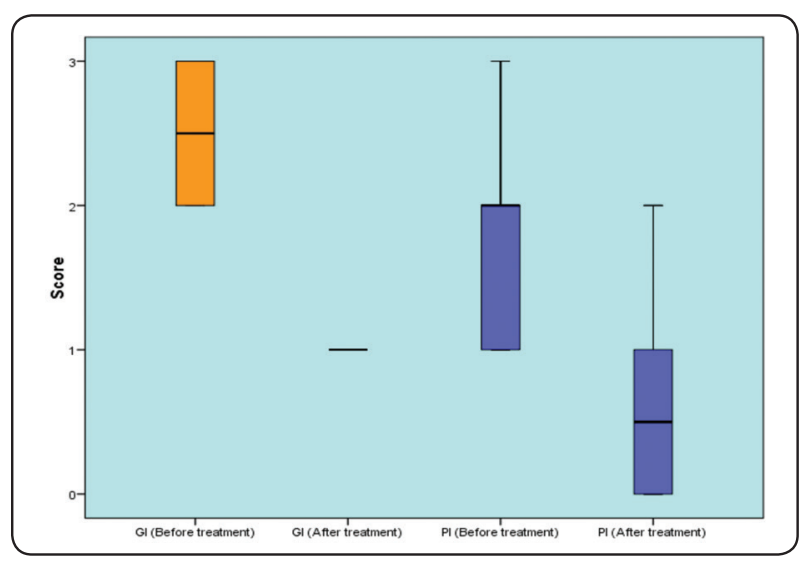

Fig. (2) Box plot representing median and range values for Plaque Index (PI) and Gingival Index (GI) before and after treatment 
TABLE (3) Descriptive statistics and results of repeated measures ANOVA test for comparison between AGE levels in the two groups and the changes within Periodontitis group

\begin{tabular}{|c|c|c|c|c|c|c|c|}
\hline \multirow{2}{*}{ AGE } & \multirow{2}{*}{ Time } & \multicolumn{2}{|c|}{$\begin{array}{l}\text { Periodontitis } \\
\qquad(\mathrm{n}=15)\end{array}$} & \multicolumn{2}{|c|}{$\begin{array}{l}\text { Control } \\
(\mathrm{n}=15)\end{array}$} & \multirow{2}{*}{$\begin{array}{c}P \text {-value } \\
\text { (Between } \\
\text { groups) }\end{array}$} & \multirow{2}{*}{$\begin{array}{c}\text { Effect size } \\
\text { (Partial Eta } \\
\text { Squared) }\end{array}$} \\
\hline & & Mean (SD) & $95 \% \mathrm{CI}$ & Mean (SD) & $95 \% \mathrm{CI}$ & & \\
\hline \multirow{4}{*}{ Serum } & Before treatment & $840.2(129.4)$ & $732-948.3$ & $476.1(119.6)$ & $376.2-576.1$ & $<0.001 *$ & 0.709 \\
\hline & After treatment & $626.6(92.9)$ & $549-704.3$ & $476.1(119.6)$ & $376.2-576.1$ & $0.014 *$ & 0.361 \\
\hline & $P$-value (Within group) & \multicolumn{2}{|c|}{$<0.001 *$} & \multicolumn{2}{|c|}{-} & & \\
\hline & $\begin{array}{l}\text { Effect size (Partial Eta } \\
\text { Squared) }\end{array}$ & \multicolumn{2}{|c|}{0.775} & \multicolumn{2}{|c|}{-} & & \\
\hline \multirow{4}{*}{ GCF } & Before treatment & $278.5(52.7)$ & $234.5-322.5$ & $172(15.5)$ & $159-184.9$ & $<0.001 *$ & 0.683 \\
\hline & After treatment & $192.2(9.6)$ & $184.2-200.2$ & $172(15.5)$ & $159-184.9$ & $0.007 *$ & 0.414 \\
\hline & $P$-value (Within group) & \multicolumn{2}{|c|}{$<0.001 *$} & \multicolumn{2}{|c|}{ - } & & \\
\hline & $\begin{array}{l}\text { Effect size (Partial Eta } \\
\text { Squared) }\end{array}$ & \multicolumn{2}{|c|}{0.785} & \multicolumn{2}{|c|}{-} & & \\
\hline
\end{tabular}

*: Significant at $P \leq 0.05$

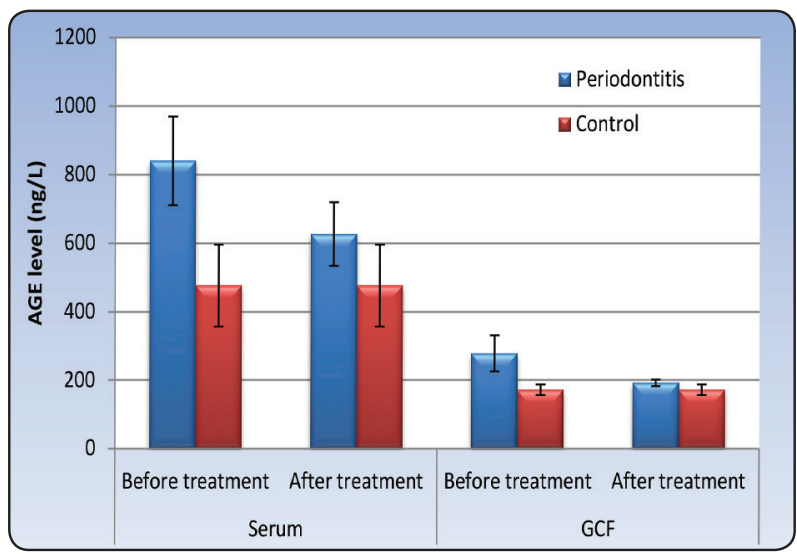

Fig. (3) Bar chart representing mean and standard deviation values for AGE levels in the two groups

Correlation between clinical parameters and Advanced Glyacation product (AGE) levels in Periodontitis group

There was no statistically significant correlation between different clinical parameters and AGE levels before or after treatment except for the correlation between PI and AGE level in GCF before treatment where there was a statistically significant inverse correlation between PI and AGE levels (Spearman's' rho “Q" $=-0.848, P$-value $=$ 0.008 ) indicating that an increase in PI is associated with a decrease in AGE GCF levels and vice versa.
Table (4) Results of Pearson's and Spearman's correlation coefficients for the correlation between clinical parameters and AGE levels in periodontitis group

\begin{tabular}{|c|c|c|c|}
\hline & Clinical parameters & $\begin{array}{c}\text { Correlation } \\
\text { coefficient }\end{array}$ & $P$-value \\
\hline \multirow{8}{*}{ 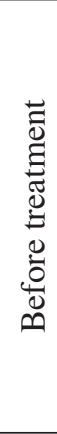 } & Serum AGE \& PD & 0.567 & 0.143 \\
\hline & Serum AGE \& CAL & 0.575 & 0.136 \\
\hline & Serum AGE \& GI & -0.655 & 0.078 \\
\hline & Serum AGE \& PI & -0.261 & 0.533 \\
\hline & GCF AGE \& PD & -0.403 & 0.323 \\
\hline & GCF AGE \& CAL & -0.152 & 0.720 \\
\hline & GCF AGE \& GI & -0.655 & 0.078 \\
\hline & GCF AGE \& PI & -0.848 & $0.008^{*}$ \\
\hline \multirow{8}{*}{ 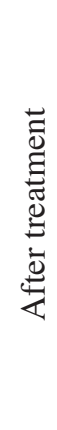 } & Serum AGE \& PD & 0.216 & 0.608 \\
\hline & Serum AGE \& CAL & -0.051 & 0.904 \\
\hline & Serum AGE \& GI & \multicolumn{2}{|l|}{$\mathrm{NC}^{\dagger}$} \\
\hline & Serum AGE \& PI & 0.183 & 0.665 \\
\hline & GCF AGE \& PD & -0.701 & 0.053 \\
\hline & GCF AGE \& CAL & -0.696 & 0.055 \\
\hline & GCF AGE \& GI & \multicolumn{2}{|l|}{$\mathrm{NC}^{\dagger}$} \\
\hline & GCF AGE \& PI & -0.091 & 0.830 \\
\hline
\end{tabular}

*: Significant at $P \leq 0.05, N^{\dagger}:$ Not Computed because GI is constant 


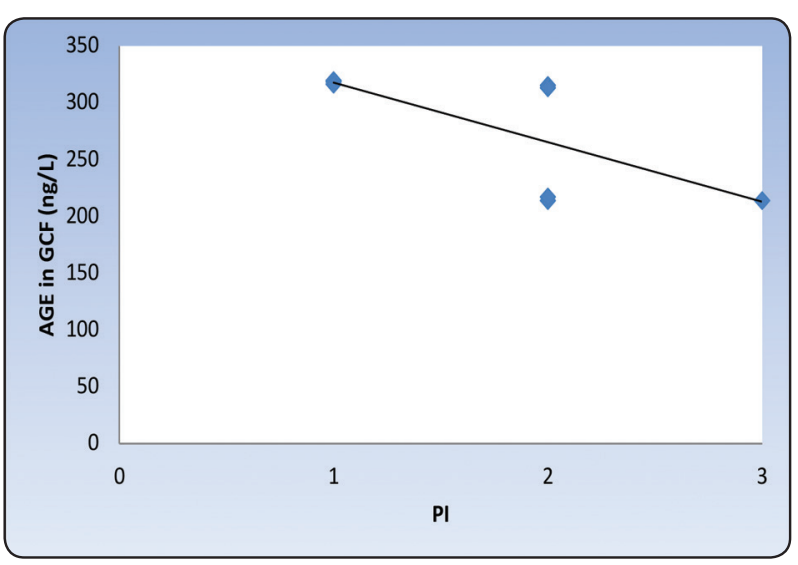

Fig. (4) Scatter diagram representing inverse correlation between Plaque Index (PI) and AGE levels in GCF before treatment in Periodontitis group

\section{DISCUSSION}

AGEs are known to affect nearly every type of cell and molecule in the body, which have pathogenic role in some conditions that causes intracellular damage and apoptosis. Recent studies have done work aiming for the treatment and prevention of related complications to some disorders including aging, kidney failure, diabetes, Alzheimer's disease, coronary artery disease, and osteoarthritis. These studies demonstrated that most of the complications especially in diabetic patients are mostly due to the accumulation of AGEs (Singh et al, 2011).

The hyper inflammatory response in periodontitis is characterized by prolonged release of neutrophil proteolytic enzymes, pro-inflammatory mediators and reactive oxygen species (ROS) (Chapple and Matthews, 2007) which play the major role in destruction of the periodontal attachment. Fusobacterium nucleatum and periodonto-pathogens are known to induce an increased intracellular production of ROS in neutrophils along with their release (Tsai, et al, 2005).

The direct evidence for the presence of AGEs in the periodontal ligament is still lacking, on the other hand, valuable approaches based on the use of periodontal cells along with genetic and bio- chemical studies in animal models and chronic periodontal patients support a potential role for protein glycation in the aetiology and severity of periodontitis (Pietropaoli et al, 2010).

Regarding both gender distributions and mean age values, the present study showed no statistically significant difference in the two groups. These results are in accordance with Zizzi et al, (2013), based on these results; age did not correlate significantly with crevicular level of AGE levels in any of their study groups; this finding was unexpected given that sample data which have shown age to be one of the decisive factors in AGEs accumulation in various organs as reported by Sato et al,2001 .

As regard to the clinical changes after treatment in periodontitis group, the present study detected a statistically significant decrease in probing depth (PD), clinical attachment level (CAL), and gingival index (GI).These results are in agreement with (Khushboo et al, 2017) and (Nobre et al, 2019).

Considering Advanced Glyacation end product (AGE) levels, the serum level either before or after treatment; the periodontitis group showed statistically significantly higher mean AGE levels than control group respectively. These results are similar to the results obtained by Takeda et al (2006) who revealed that serum AGEs levels were significantly associated with deterioration of periodontitis, accordingly, they suggested AGEs as a useful biomarkers that reflect periodontal deterioration. Up to author's knowledge, there are very few studies that explored the serum level of AGEs.

Tsai, et al, (2005) detected higher levels of ROS in saliva and gingival crevicular fluid of periodontitis patients when compared with healthy controls.

Similarly, in the study performed by $\mathbf{L i}$ et $\mathbf{a l}$, (2014), the periodontal ligaments fibroblasts treated with AGE-BSA were deformed and expressed higher levels of RAGE and caspase 3.The cell 
viability assays and flow cytometry indicated that AGE-BSA reduced cell viability and increased apoptosis. They concluded that the changes in periodontal ligaments fibroblasts induced by AGEBSA may explain how AGE-RAGE participates in and exacerbates periodontium destruction.

Due to the potential diagnostic importance of gingival crevicular fluid, the present study focused the light on the crevicular level of AGEs especially that, up to author's knowledge, no other studies explored the crevicular level of AGEs. The current study detected a statistically significantly higher mean AGE levels in the periodontitis group than control group either before or after treatment, respectively. This can be explained by accumulation of AGEs in periodontal tissues and their role in the microvascular alterations as found in diabetic patients as reported by Schmidt et al,(1996), where AGEs are known to bind irreversibly to collagen forming macro-molecules which accumulate in the basement membranes of endothelial cells resulting in increased basement membrane thickness and alteration in the normal homeostatic transport across the membrane as mentioned by Seppala \& Ainamo (1994).

Recently, Allen et al. in 2011 proposed that the hyperactive neutrophils, which possibly activated within the periodontium, maybe an important source of reactive oxygen species which in turn activate the pro-inflammatory pathways and then promote insulin resistance in patients with periodontitis and diabetes.

The increased oxidative stress plays a major role in elevated lipid peroxidation which in turn may have a pro-inflammatory effect. Bastos et al. in 2012 demonstrated higher levels of GCF markers of lipid peroxidation in diabetes patients which correlated with both the clinical parameters of periodontitis and levels of inflammatory mediators.

On the same hand, AGEs were found to have an adverse effect on bone collagen at the cellular level and this may explain the alterations in bone metabolism (Vashisht, 2001) and (Monnier et al, 2005).

In addition, Glycation of the bone collagen was found to affect the bone turnover, inducing reduced bone formation which in turn, reduces osteoblastic differentiation and ECM production (Gunczler et al, 2001) and (McCarthy et al, 2001).

Later on, AGEs were found to participate in the pathogenic mechanisms of periodontitis independently of the mechanisms underlying their accumulation (Pietropaoli et al, 2010).

Regarding the changes after treatment in Periodontitis group, whether in serum or GCF, the current study revealed a statistically significant decrease in AGEs level after treatment respectively. This can be explained by the effect of periodontal treatment which not only reduces the oral inflammation, but also decreases the systemic levels of different inflammatory makers such as IL-6, TNF- $\alpha$, and C-reactive proteins (CRP), indicating that periodontal diseases induce systemic alterations beyond the local periodontal environment (D'Aiuto, et al, 2004).

The present study detected no statistically significant correlation between different clinical parameters and AGE levels before or after treatment in periodontitis patients.

Based on the observations of included studies, and on the results of the current study, it can be concluded that GCF is an effective and efficient prognostic tool by means of analysis of variations in constituents before and after periodontal therapy (Stuti et al, 2018).

Accordingly, crevicular level AGE can be used as a biomarker for the diagnosis and prognosis of periodontitis.

Finally, it is recommended to conduct larger and long-term studies in the future, as one of the 
limitations of the current study is the small sample size. The small period of follow up is another limitation.

\section{CONCLUSION}

Crevicular level of AGE can be used as a noninvasive diagnostic and prognostic biomarker for periodontitis.

\section{REFRENCES}

- Abramson JH. (1966). The Cornell Medical Index as an epidemiological tool Am J Public Health N; 56:287-298.

- Allen EM, Matthews, O'Connor R, O'Halloran, Chapple IL. (2009). Periodontitis and type 2 diabetes: is oxidative stress the mechanistic link? Scott Med J; 54:41-7.

- $\quad$ Allen, E. M., Matthews, J. B., DJ, O. H., Grif-fiths, H. R. \& Chapple, I. L. (2011)/ Oxidativeand inflammatory status in Type 2 diabetes patients with periodontitis. Journal of ClinicalPeriodontology38: 894-901

- Bastos, A. S., Graves, D. T., Loureiro, A. P.,Rossa Junior, C., Abdalla, D. S., FaulinTdo, E., Olsen Camara, N., Andriankaja, O.M. \& Orrico, S. R. (2012). Lipid peroxidationis associated with the severity of periodontal disease and local inflammatory markers in patients with type 2 diabetes. Journal of Clinical Endocrinology and Metabolism 97, E1353-1362.

- Chapple IL, Matthews JB. (2007). The role of reactive oxygen and antioxidant species in periodontal tissue destruction. Periodontol2000; 43:160-232.

- Elaheh Foroumandi and Abdolhassan Kazemi. (2018), Review on Advanced Glycation End Products and the Progress of Chronic Disorders *Curr TrendsBiomedical Eng \& Biosci.; 12(5): 555846

- F. D'Aiuto, M. Parkar, G. Andreou, et al. (2004). Periodontitis and systemic inflammation: control of the local infection is associated with a reduction in serum inflammatory markers J Dent Res, 83 , pp. 156-160

- Goldin A, Beckman JA, Schmidt AM, Creager MA (2006) Advanced glycation end products: sparking the development of diabetic vascular injury. Circulation 114(6): 597-605.

- Griffiths GS.(2003).Formation, collection and significance of gingival crevice fluid. Periodontol 2000; 31:32-42.
- Gunczler, P, Lanes, R, Paoli, M, Martinis, R, Villaroel, O, Weisinger, JR. (2001). Decreased bone mineral density and bone formation markers shortly after diagnosis of clinical Type 1 diabetes mellitus. J Pediatr Endocrinol Metab. ; 14: 525-8.

- Khushboo Goel, Shaili Pradhan, and Madhur Dev Bhattarai. (2017). Effects of nonsurgical periodontal therapy in patients with moderately controlled type 2 diabetes mellitus and chronic periodontitis in Nepalese population. Clin Cosmet Investig Dent.; 9: 73-80.

- $\quad$ Li DX, T.Z. Deng, J. Lv, and J. Ke , Braz. (2014). Advanced glycation end products (AGEs) and their receptor (RAGE) induce apoptosis of periodontal ligament fibroblasts. J Med Biol Res. 47(12): 1036-1043.

- McCarthy, AD, Etcheverry, SB, Bruzzone, L, Lettierri, G, Barrio, DA, Cortizo, AM. (2001). Non-enzymatic glycosylation of a type I collagen matrix: Effects on osteoblastic development and oxidative stress.BMC Cell Biol. 2: 16-21.

- McDevitt MJ, Wang HY, Knobelman C, et al. (2000). Interleukin-I genetic association with periodontitis in clinical practice. J. Periodontology; 71: 156- 63.

- Monnier, VM, Mustata, GT, Biemel, KL et al. (2005). Cross-linking of the extracellular matrix by the Maillard reaction in aging and diabetes: An update on "a puzzle nearing resolution”. Ann N Y Acad Sci. 1043: 533- 44.

- Nobre ÁVV, Pólvora TLS, Silva LRM, Teles VO, Villafuerte KV, da Motta RJG, Fortes JHP, Silva GA, Ranieri ALP, de Macedo LD, Morejon KML, da Fonseca BAL, Tirapelli C, Saraiva MCP, Taba M Jr, Lourenço AG, Motta ACF. (2019). Effects of non-surgical periodontal therapy on clinical and immunological profile and oral colonization of Candida spp in HIV-infected patients with chronic periodontitis. J Periodontol.Feb;90(2):167-176.

- $\quad$ Pietropaoli D., Tatone C., D’Alessandro A.M. and Monaco A. (2010). Possible involvement of advanced Glycation End Products in periodontal diseases. Int. J. ImmunopathoI. PharmacoI. Vol. 23, no. 3, 683-69.

- Sato Y, Kondo T, Ohshima T. (2001). Estimation of age of human cadavers by immunohistochemical assessment of advanced glycation end products in the hippocampus. Histopathology;38: 217-220.

- Schmidt A, Weidman E, Lalla E, et al. (1996). Advanced glycation end products (AGEs) induce oxidant stress in the gingiva: a potential mechanism underlying accelerated 
periodontal disease associated with diabetes. J Periodontol Res.; 31:508-15.

- $\quad$ Schwarz F, Bieling K, Latz T, Nuesry E, Becker J (2006). Healing of intrabony peri- implantitis defects following application of a nanocrystalline hydroxyapatite (Ostim) or a bovine-derived xenograft (Bio-Oss) in combination with a collagen membrane (Bio-Gide). A case series. J Clin Periodontol 33: 491-499. S

- Seppala B, Ainamo J. (1994). A site-by-site follow-up study on the effect of controlled versus poorly controlled insulin-dependent diabetes mellitus. J Clin Periodontol. ;21: 161-5.

- Shoelson SE, Lee J, Goldfine AB (2006). Inflammation and insulin resistance. The Journal of clinical investigation 116(7): 1793-801.

- Singh R, Barden A, Mori T, Beilin L (2011) Advanced glycation endproducts: a review. Diabetologia 44(2): 129146.

- $\quad$ Stuti Gupta, Shivjot Chhina, Sachit Anand Arora. (2018). A systematic review of biomarkers of gingival crevicular fluid: Their predictive role in diagnosis of periodontal disease status. J Oral Biol Craniofac Res. 8(2) 98-104.

- $\quad$ Szczepanski M, Kamianowska M, Skrzydlewska E. (2007). The influence of hyperglycemia on oxidationreduction reaction of human umbilical vein endothelial cells. Pol Merkur Lekarski; 23:246- 50.
- Takeda M, Ojima M, Yoshioka H, Inaba H, Kogo M, Shizukuishi S, Nomura M, Amano A. (2006). Relationship of serum advanced glycation end products with deterioration of periodontitis in type 2 diabetes patients. $\mathrm{J}$ Periodontol. Jan; 77(1):15-20.

- Tsai CC, Chen HS, Chen SL, Ho YP, Ho KY, Wu YM, Hung CC.(2005). L Lipid peroxidation: a possible role in the induction and progression of chronic periodontitis. $\mathrm{J}$ Periodontal Res; 40:378-84

- Tu“ter G, Serdar M, Kurtisx B, et al. (2010). Effects of scaling and root planing and subantimicrobial dose doxycycline on gingival crevicular fluid levels of matrix metalloproteinase-8, -13 and serum levels of HsCRP in patients with chronic periodontitis. J Periodontol; 81:11321139.

- Vashisht, D, Gibson, GJ, Khoury, JI, Schaffler, MB, Kimura, J, Fyhrie, DP. (2001). Influence of non enzymatic glycation on biomechanical properties of cortical bone. Bone. ; 28: 195- 201.

- Zizzi A, Tirabassi G, Aspriello SD, Piemontese M, Rubini C, Lucarini G. (2013). Gingival advanced glycation end-products in diabetes mellitus-associated chronic periodontitis: an immunohistochemical study. J Periodont Res; 48: 293-301. 